Proceeding Series of the Brazilian Society of Computational and Applied Mathematics

\title{
Uma Metodologia Baseada Na Identificação Fuzzy Sobre Um Método Híbrido Para Modelos de Previsão em Série Temporal
}

\author{
José Gracildo de Carvalho Júnior ${ }^{1}$
}

Programa de Pós-Graduação em Engenharia Elétrica; Universidade Federal do Pará; Belém, Pará, Brasil

Carlos Tavares da Costa Júnior ${ }^{2}$

Programa de Pós-Graduação em Engenharia Elétrica; Universidade Federal do Pará; Belém, Pará, Brasil

João Caldas do Lago Neto ${ }^{3}$

Grupo de Otimização e Sistemas Fuzzy; Universidade Federal do Amazonas; Manaus, Amazonas, Brasil

Resumo. Este trabalho apresenta um método de previsão híbrido, com concepção e aprimoramento pelo uso da teoria dos conjuntos fuzzy, aliada à metodologia clássica de séries temporais. Em comparação com métodos de previsão consolidados na literatura, os resultados apresentados pelo método proposto foram considerados plenamente satisfatórios, face o baixíssimo erro retornado.

Palavras-chave. Conjuntos fuzzy, Método fuzzy de identificação, Série temporal fuzzy, Previsão fuzzy, Erro de previsão.

\section{Introdução}

\subsection{Motivação e objetivos}

Metodologias capazes de prever variáveis de comportamento rotineiros, geralmente imprevisíveis (ver, [19]), como fenômenos da engenharia, medicina, biologia, física, etc., têm fomentado uma quantidade significativa de pesquisas científicas. Dentre os diversos fatores com efeitos diretos na capacidade preditiva dos métodos de previsão, é senso comum que a identificação correta dos parâmetros estruturais, se torna uma das etapas fundamentais e mais importante do processo de modelagem matemática, tal como, é amplamente discutido em [2]. Neste contexto, a utilização de técnicas de previsão temporal que consideram metodologias híbridas, como por exemplo, série temporal fuzzy, para muitos estudiosos surge como uma alternativa promissora, pois apresenta eficiência amplamente comprovada, diante de uma infinidade de propostas estabelecidas na literatura. Desta forma, este estudo busca propor uma estratégia de previsão fuzzy, que torne possível estabelecer uma rotina de modelagem temporal, onde o erro global inerente ao modelo de previsão seja minimizado, por meio da determinação eficiente dos parâmetros estruturais da série temporal fuzzy.

\footnotetext{
${ }^{1}$ gracildo@ufpa.br

${ }^{2}$ cartav@ufpa.br

3 jcaldas@ufam.edu.br
} 


\subsection{Estado da arte}

Originalmente proposto por [27], o método de previsão conhecido como série temporal fuzzy, há bastante tempo vem suscitando uma quantidade expressiva de propostas metodológicas, dentre as quais muitas são concebidas combinadas à inteligência artificial, por exemplo, o método de otimização por enxame de partículas [16], algoritmos genéticos [5] e redes neurais artificiais [1]. Neste contexto, os métodos de previsão constituídos por séries temporais, que incorporam em seus algoritmos a teoria de conjuntos fuzzy, que foi estabelecida por [32], passaram a receber um destaque especial por grande parte dos pesquisadores. Desta forma, modelos híbridos de previsão fuzzy são utilizados nas mais diversas áreas do conhecimento humano, tais como, atividade solar [1], carga de energia elétrica $[12,15,21,26,29]$, demanda de petróleo [25], produção de arroz [3], índice de ações chinês $[8,9,28]$, funcionamento de turbinas em centrais nucleares [4], índice TAIEX [22,23], volume de tráfego de navios no estreito de Istambul [7], velocidade diária dos ventos [30], consumo de diesel e energia elétrica [20].

\section{Propósito}

\subsection{Importância do trabalho}

A busca pela otimização global na identificação do modelo de previsão fuzzy, que implica numa maior acurácia do modelo estimado, tem surgido como uma necessidade crescente no processo de modelagem temporal. Neste contexto, este trabalho contribui, para a solução de problemas em uma demanda existente no mundo real, sobre tudo, com uma forte e expressiva corrente científica que busca propor ferramentas com capacidade suficiente, para melhorar o algoritmo funcional de estimação paramétrica em série temporal.

\subsection{Destaques desta pesquisa}

Com a aplicação do algoritmo proposto neste trabalho sobre dados fuzzificados, se torna possível obter um modelo inicial, que captura o padrão das características aleatórias de forma eficiente, independente do baixo ou alto grau de variabilidade inerente à base de dados utilizados. Assim, a proposta desta pesquisa origina-se sobre um modelo temporal preliminar, e, com a utilização da função de autocorrelação estendida, aliada a um sistema fuzzy de funções de pertinências triangulares, produz um modelo de previsão com estrutura híbrida, que suscita um método de modelagem fuzzy temporal otimizado computacionalmente, com capacidade preditiva significativa e de caráter inédito na literatura.

\section{Métodos}

Definição 1. Seja uma variável aleatória $Z(t)$, onde $t=0 ; 1 ; 2 ; \ldots ; n$ é um conjunto dos números inteiros, tal que, $U$ representa o universo de discurso onde os conjuntos fuzzy $f_{i}(t)(i=0 ; 1 ; 2 ; \ldots ; n)$ são definidos $[26,28]$. Caso $F(t)$ represente uma coleção de $f_{1}(t)$; $f_{2}(t) ; \ldots ; f_{n}(t)$, então, $F(t)$ é considerada uma série temporal fuzzy sobre a variável $Z(t)$.

Definição 2. Caso $F(t)$ seja resultante de $F(t-1)$, então se pode representar esta relação como $F(t-1) \rightarrow F(t)$. Neste caso, esta relação fuzzy pode ser dada segundo $[3,10,21,26,28]$, como $F(t)=F(t-1) \circ U(t-1, t)$, onde $U(t-1, t)$ indica a relação fuzzy entre $F(t)$ e $F(t-1)$, e ainda, "o" representa um operador de composição máximo-mínimo (ver, [6]). 
Definição 3. Segundo [28], representando $F(t-1)$ por $A_{i}$ e $F(t)$ por $A_{j}$, então, a relação entre $F(t)$ e $F(t-1)$, consequentemente pode ser considerada uma relação lógica $A_{i} \rightarrow A_{j}$. Definição 4. Seja $F(t)$ uma série temporal fuzzy. Caso exista um componente de sazonalidade em $F(t)$, então um modelo de previsão via série temporal fuzzy sazonal de primeira ordem pode ser estimado e, segundo ainda [26], este modelo é representado por $F(t-s) \rightarrow F(t)$, onde " $s$ " indica o período sazonal observado.

Definição 5. Define-se por função de autocorrelação estendida fuzzy média aleatória (EACF fuzzy- $R A$ ), para a média da EACF no intervalo $u_{i}$, correspondente ao conjunto fuzzy triangular $A_{i}$ ativado pela pertinência máxima, durante o processo de fuzzificação. Assim, são gerados " $n$ " pontos dentro do suporte do conjunto $A_{i}$, e, posteriormente é calculada a função EACF fuzzy- $R A$, no intervalo $u_{i}$ específico para compor a série temporal fuzzy $F_{i}(t)$, onde, $1 \leq i \leq m$ e $1 \leq t \leq T$, como sendo o conjunto de valores obtidos. A função média aleatória fuzzy resultante deste processo é representada pela Equação (1).

$$
W_{t, k, j}^{R A}(h ; b)=F_{t}^{R A}(b)-\widetilde{\phi}_{1}^{R A}(b) F_{t-1}^{R A}(b)-\cdots-\widetilde{\phi}_{k}^{R A}(b) F_{t-k}^{R A}(b),
$$

onde, $h=1 ; 2 ; \ldots r, k \geq 0, j \geq 0, b=1 ; 2 ; \ldots ; B$, tal que, $B$ é o número de amostras independentes e $\widetilde{\phi}_{r ; k}^{R A}(b)$, são obtidos de forma iterativa, de acordo como é descrito em [11].

\subsection{Medida de desempenho para avaliação do modelo de previsão fuzzy}

Mediante à identificação do modelo de previsão, uma avaliação dos erros deste modelo ajustado, necessariamente deve ser realizada, como defendem $[14,18]$. Neste sentido, a medida de acurácia do erro de previsão, conhecida por escala de erro médio absoluto (MASE), surge como a mais indicada segundo [18]. Então, como em [13], neste estudo será adotada a Equação (2).

$$
M A S E=\text { Mean }\left|\frac{e_{t}}{\frac{1}{n-1} \sum_{t=2}^{n}\left|Y_{t}-Y_{t-1}\right|}\right|,
$$

onde, $e_{t}$ indica o erro no instante $t$, tal que, $t=1, \ldots, n, n$ é o número de dados da série temporal e $Y_{t}$ e $Y_{t-1}$, indicam os valores da série no instante $t$ e $t-1$, respectivamente.

\subsection{Algoritmo de previsão fuzzy proposto}

Nesta seção, o algoritmo de previsão fuzzy, a ser implementado sobre a série temporal, será apresentado na forma de rotina descrita passo a passo.

Etapa 1: Coletar os dados históricos de interesse para compor a série temporal;

Etapa 2: Definir o universo de discurso $U$, onde $U=\left[Z_{(\min )}-Z_{1} ; Z_{(\max )}+Z_{2}\right]$, tal que, $Z_{(\min )}$ e $Z_{(\max )}$, são os valores mínimo e máximo da série de dados, respectivamente; $Z_{1}$ e $Z_{2}$ são dois números positivos como observados em [9,10];

Etapa 3: Particionar o universo $U$ em " $m$ " conjuntos fuzzy de mesmo comprimento como em [3]. Segundo [24], $m \in[5 ; 9]$, logo, a média $m=7$ é um valor ideal a ser adotado;

Etapa 4: Fuzzificar os dados construindo $m$ conjuntos fuzzy $A_{i}$, onde $i=1 ; 2 ; \ldots ; m$, determinados no passo anterior, utilizando a definição de número fuzzy triangular;

Etapa 5: Ativar os conjuntos fuzzy construídos no passo anterior, pela pertinência máxima de cada valor em relação à $A_{i}$, e posteriormente, indicar as relações fuzzy lógicas. 
Etapa 6: Gerar uma série temporal com 1000 pontos aleatórios no intervalo $u_{i}$, associado $\operatorname{com} A_{i}$ ativado no passo anterior. Calcular a função de autocorrelação amostral (fac) dentro dos $u_{i}^{\prime} s$. Obter a fac-média aleatória $(f a c-M A)$ em cada $u_{i}$. Calcular a EACF das $f a c-M A^{\prime} s$ nos $u_{i}^{\prime} s$ resultantes. E, então, identificar o modelo fuzzy;

Etapa 7: Gerar previsões pelo modelo de série temporal fuzzy, que foi identificado no passo anterior. Medir a acurácia do modelo identificado mediante o valor do MASE.

\section{Resultados}

Nesta seção, a rotina de identificação e previsão fuzzy que está sendo proposta neste trabalho, será avaliada em um estudo de caso, utilizando um modelo de previsão via série temporal fuzzy. Uma base de dados sobre carga de energia elétrica disponível na literatura científica (ver, [26]), será utilizada na avaliação do algoritmo de previsão fuzzy proposto.

\subsection{Previsão fuzzy para a carga de energia elétrica de curto prazo}

De acordo com os passos do algoritmo de previsão, descritos na seção 3, inicialmente determina-se $U=[21340 ; 43000]$, e escolhe-se $m=7$, pois, segundo a teoria defendida por [24], sete partições de $U$ é o mais adequado. Para $A_{i}$, onde $i=1 ; 2 ; \ldots ; 7$, e utilizando a definição de número fuzzy triangular discutida em [3], onde os triângulos são isósceles de vértices dados por $a_{i} ; b_{i} ; c_{i}$, e o intervalo de suporte de cada conjunto fuzzy é definindo por $u_{i}=\left[a_{i} ; c_{i}\right]$. Os intervalos de suporte dos conjuntos fuzzy são: $u_{1}=[21340 ; 26760]$, $u_{2}=[24050 ; 29460], u_{3}=[26760 ; 32170], u_{4}=[29460 ; 34880], u_{5}=[32170 ; 37590], u_{6}=[34880 ;$ 40290] e $u_{7}=[37590 ; 43000]$. Após o processo de fuzzificação ser finalizado (Etapa 5), na Etapa 6 a identificação do modelo foi realizada e os resultados da função $W_{t, k, j}^{R A}$ são observados nas Tabelas (1 e 2). O modelo ótimo identificado (probabilidade de significância $<\alpha=10 \%$ ), é o modelo ARMA de parâmetros $\operatorname{AR}(5)$ e MA(3) (0,086 na Tabela 2).

Tabela 1: Resultados da função $W_{t, k, j}^{R A}$ para a carga de energia elétrica.

\begin{tabular}{crrrrrr}
\hline Lag & MA(0) & MA(1) & MA(2) & MA(3) & MA(4) & MA(5) \\
\hline AR(0) & $-0,405$ & 0,009 & $-0,164$ & 0,174 & $-0,026$ & $-0,114$ \\
AR(1) & $-0,379$ & $-0,027$ & $-0,125$ & 0,172 & $-0,022$ & $-0,035$ \\
AR(2) & $-0,452$ & $-0,442$ & $-0,366$ & 0,029 & $-0,075$ & $-0,011$ \\
AR(3) & $-0,136$ & 0,035 & $-0,393$ & $-0,085$ & $-0,106$ & $-0,228$ \\
AR(4) & 0,090 & $-0,011$ & $-0,171$ & $-0,094$ & 0,033 & $-0,214$ \\
AR(5) & 0,023 & $-0,171$ & $-0,136$ & $-0,313$ & $-0,045$ & $-0,079$ \\
\hline
\end{tabular}

Tabela 2: Probabilidade de significância para os resultados da função $W_{t, k, j}^{R A}$ na Tabla 1.

\begin{tabular}{cllllll}
\hline Lag & MA(0) & MA(1) & MA(2) & MA(3) & MA(4) & MA(5) \\
\hline AR(0) & 0,006 & 0,956 & 0,328 & 0,309 & 0,882 & 0,515 \\
AR(1) & 0,010 & 0,870 & 0,481 & 0,301 & 0,909 & 0,868 \\
AR(2) & 0,002 & 0,003 & 0,020 & 0,865 & 0,765 & 0,965 \\
AR(3) & 0,368 & 0,820 & 0,012 & 0,643 & 0,618 & 0,196 \\
AR(4) & 0,554 & 0,946 & 0,460 & 0,678 & 0,882 & 0,260 \\
AR(5) & 0,880 & 0,308 & 0,592 & 0,086 & 0,826 & 0,743 \\
\hline
\end{tabular}

Para que o modelo seja considerado válido, dentre outras condições, a série temporal que originou este modelo deve ser estacionária. O teste de Dickey-Fuller da raiz unitária (ver, [17]), indica que a série defuzzificada da carga de energia elétrica possui comportamento estacionário ( $\mathrm{p}$-valor $=0,001<\alpha=10 \%$ ). O modelo otimizado computacionalmente após a identificação foi o $\operatorname{FSARIMA}(5 ; 1 ; 1)(1 ; 0 ; 1)_{36}$, onde a estatística de teste $t$-Student indicou os parâmetros $\mathrm{AR}(2)$ e $\mathrm{AR}(3)$, deste modelo como não significativos, isto é, p-valor $=0,316$ e 0,201 (ambos $>10 \%$ ), respectivamente. Portanto, o modelo de previsão fuzzy irá excluir estes parâmetros, diferentemente dos demais coeficientes que serão mantidos no modelo. Como um componente de sazonalidade $(s=36)$ está integrado ao modelo, o teste Qui-quadrado comprovou sua significância estatística (p-valor $=0,032<\alpha=0,10$ ). 


\section{Discussões}

Finalmente, na Etapa 7 do algoritmo de previsão, o MASE obtido assumiu valor de 0,14 (Tabela 3). Como o MASE é uma métrica de erro absoluto $(\geq 0)$, se pode aceitar que o modelo foi bem identificado. E ainda, em comparação com outros métodos de previsão vistos na Tabela 3, a eficiência do método de previsão proposto é comprovada. A eficiência do método de identificação proposto se torna possível de ser ratificada, com o auxílio da Figura (1), face uma sobreposição entre os valores reais e de previsão pelo modelo proposto.

Tabela 3: Comparação do MASE obtido por vários métodos para carga de energia elétrica.

\begin{tabular}{llc}
\hline Fonte & Modelo & MASE \\
\hline Sadaei et al. (2014) & Método OTS-SVR & 1,09 \\
Sadaei et al. (2014) & Método PSO-ARIMA & 0,71 \\
Sadaei et al. (2014) & Método LS-ARIMA & 0,51 \\
Sadaei et al. (2014) & Método REWFTS-Model 1 & 0,60 \\
Sadaei et al. (2014) & Método Sadaei et al. & 0,37 \\
Método Proposto & FSARIMA(5,1,1)(1,0,1) 36 & 0,14 \\
\hline
\end{tabular}

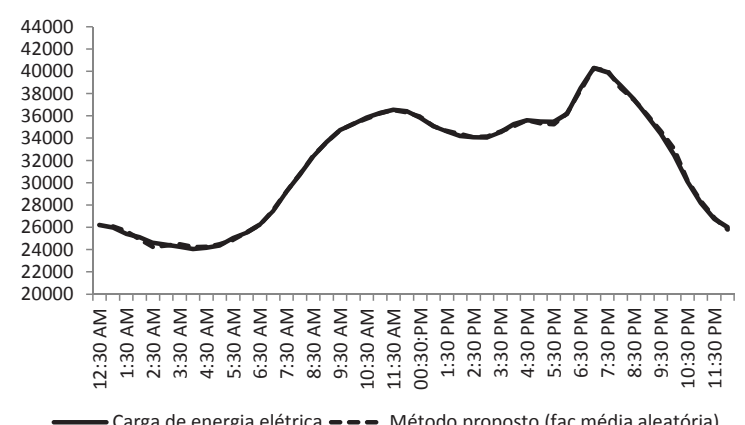

Figura 1: Valor atual e previsões (método proposto) para carga de energia elétrica.

Adicionalmente, foram construídos intervalos de confiança fuzzy (ver, [31]) com altíssimo grau de confiabilidade (99\%). Mediante os resultados de 6 projeções (12:30 PM a 03:00 AM) realizadas pelo modelo proposto e observadas na Tabela (4), é possível notar valores da carga de energia elétrica, que pertencem aos intervalos de confiança. Este fato ratifica novamente que o modelo de previsão fuzzy proposto foi eficientemente identificado.

Tabela 4: Intervalo de confiança fuzzy para a carga de energia elétrica.

\begin{tabular}{cccccc}
\hline $\begin{array}{c}\text { Tempo } \\
\text { horas) }\end{array}$ & $\begin{array}{c}\text { Projeção da carga } \\
\text { de energia elétrica }(\mathrm{MW})\end{array}$ & $\begin{array}{c}\text { Conjunto } \\
\text { fuzzy }\end{array}$ & $\begin{array}{c}\text { Intervalo } \\
\text { fuzzy }\left(u_{i}\right)\end{array}$ & I.C $\left(\widetilde{\mu}_{h}^{L} ; 99 \%\right)$ & I.C $\left(\widetilde{\mu}_{h}^{U} ; 99 \%\right)$ \\
\hline 12:30 PM & $26.859,60$ & A2 & {$[24.050 ; 29.460]$} & {$[23.760,12 ; 35.168,45]$} & {$[29.175,91 ; 40.581,23]$} \\
01:00 AM & $28.641,50$ & A3 & {$[26.760 ; 32.170]$} & {$[23.760,12 ; 35.168,45]$} & {$[29.175,91 ; 40.581,23]$} \\
$01: 30$ AM & $31.736,40$ & A4 & {$[29.460 ; 34.880]$} & {$[23.760,12 ; 35.168,45]$} & {$[29.175,91 ; 40.581,23]$} \\
02:00 AM & $34.892,10$ & A5 & {$[32.170 ; 37.590]$} & {$[23.760,12 ; 35.168,45]$} & {$[29.175,91 ; 40.581,23]$} \\
$02: 30$ AM & $37.585,80$ & A6 & {$[34.880 ; 40.290]$} & {$[23.760,12 ; 35.168,45]$} & {$[29.175,91 ; 40.581,23]$} \\
03:00 AM & $39.733,10$ & A7 & {$[37.590 ; 43.000]$} & {$[23.760,12 ; 35.168,45]$} & {$[29.175,91 ; 40.581,23]$} \\
\hline
\end{tabular}

Em relação às suposições básicas de ruído branco em uma série temporal, de acordo ao que defende [14]. A partir do padrão aleatório apresentado pelos erros, se torna possível aceitar que os erros de previsão apresentam variância constante e são independentes entre si. Consequentemente, quanto à distribuição normal dos erros de previsão que foram gerados pelo modelo FSARIMA $(5 ; 1 ; 1)(1 ; 0 ; 1)_{36}$, o teste de normalidade de Anderson Darling (AD) forneceu valor de estatística igual a 0,501, com probabilidade de significância de 0,198. Como o p-valor $=0,198>\alpha=10 \%$, então, os erros produzidos pelo modelo de previsão fuzzy proposto neste trabalho, seguem uma função densidade de probabilidade normal. 


\section{Conclusões}

Neste estudo, foi estabelecido um novo algoritmo de previsão fuzzy, com o intuito de reduzir os erros associados ao modelo resultante, mediante uma identificação eficiente dos parâmetros estruturais. Para isto, combinou-se a metodologia de conjuntos fuzzy à função de autocorrelação estendida e se otimizou o processo de identificação, com auxilio de testes computacionais suscitando um modelo de previsão fuzzy com acurácia elevada. O erro de previsão retornado pelo modelo proposto foi necessariamente baixo, atendendo as exigências estabelecidas na literatura em constituir um padrão de ruído branco exigido.

\section{Referências}

[1] M. Abdollahzade, A. Miranian, H. Hassani and H. Iranmanesh, A new hybrid enhanced local linear neuro-fuzzy model based on the optimized singular spectrum analysis and its application for nonlinear and chaotic time series forecasting, Information Sciences., vol. 295, 107-125, (2015).

[2] L. A. Aguirre, Introdução à identificação de sistemas: técnicas lineares e não-lineares aplicadas a sistemas reais, Editora UFMG, Edição 3 (revisada e ampliada), Belo Horizonte, (2007).

[3] S. Askari and N. Montazerin, A high-order multi-variable fuzzy time series forecasting algorithm based on fuzzy clustering, Expert Systems with Applications., vol. 42, 2121-2135, (2015).

[4] P. Baraldi, F. D. Maio, D. Genini and E. Zio, Reconstruction of missing data in multidimensional time series by fuzzy similarity, Applied Soft Computing., vol. 26, 1-9, (2015).

[5] E. Bas, V. R. Uslu, U. Yolcu and E. Egrioglu, A modified genetic algorithm for forecasting fuzzy time series, Applied Intelligence., vol. 41, 453-463, (2014).

[6] R. Boukezzoula, S. Galichet and L. Foulloy, MIN and MAX Operators for Fuzzy Intervals and Their Potential Use in Aggregation Operators, IEEE Trans Fuzzy Syst., vol. 15, 1135-1144, (2007).

[7] E. Bulut, Modeling seasonality using the fuzzy integrated logical forecasting (FILF) approach, Expert Systems with Applications., vol. 41, 1806-1812, (2014).

[8] Q. Cai, D. Zhang, W. Zheng and S. C. H. Leung, A new fuzzy time series forecasting model combined with ant colony optimization and auto-regression, Knowledge-Based Systems., vol. $74,61-68,(2015)$.

[9] M. Y. Chen, and B. T. Chen, A hybrid fuzzy time series model based on granular computing for stock price forecasting, Information Sciences., vol. 294, 227-241, (2015).

[10] M. Y. Chen, A high-order fuzzy time series forecasting model for internet stock trading, Future Generation Computer Systems., vol. 37, 461-467, (2014).

[11] J. D. Cryer and K. S. Chan, Time Series Analysis - With Applications in R, Springer, Cap. 6 , vol. 2, (2008).

[12] Y. Dong, J. Wang, H. Jiang and J. Wu, Short-term electricity price forecast based on the improved hybrid model, Energy Conversion and Management., vol. 52, 2987-2995, (2011).

[13] O. Duru and E. Bulut, A non-linear clustering method for fuzzy time series: Histogram damping partition under the optimized cluster paradox, Applied Soft Computing., vol. 24, $742-748,(2014)$.

[14] O. Duru, A fuzzy integrated logical forecasting model for dry bulk shipping index forecasting: An improved fuzzy time series approach, Expert Systems with Applications., vol. 37, 53725380, (2010). 
[15] R. Efendi, Z. Ismail and M. M. Deris, A new linguistic out-sample approach of fuzzy time series for daily forecasting of Malaysian electricity load demand, Applied Soft Computing., vol. 28, 422-430, (2015).

[16] E. Egrioglu, PSO-based high order time invariant fuzzy time series method: Application to stock exchange data, Economic Modelling., vol. 38, 633-639, (2014).

[17] S. Hylleberg, Tests for seasonal unit roots general to specific or specific to general?, Journal of Econometrics., vol. 69, 5-25, (1995).

[18] R. J. Hyndman and A. B. Koehler, Another look at measures of forecast accuracy, International Journal of Forecasting., vol. 22, 679-688, (2006).

[19] H. Kantz and T. Schreiber, Nonlinear Time Series Analysis, Cambridge University Press, Cambridge, Cap. 2, vol. 7, (2004).

[20] J. C. Lago Neto, C. T. Costa Junior, S. D. B. Bitar and W. Barra Junior, Forecasting of energy and diesel consumption and the cost of energy production in isolated electrical systems in the amazon using a fuzzification process in time series models, Energy Policy., vol. 39, 4947-4955, (2011).

[21] W. J. Lee and J. Hong, A hybrid dynamic and fuzzy time series model for mid-term power load forecasting, International Journal Electrical Power and Energy Systems., vol. 64, 1057-1062, (2015).

[22] W. Lu, X. Chen, W. Pedrycz, X. Liu and J. Yang, Using interval information granules to improve forecasting in fuzzy time series, International Journal of Approximate Reasoning., vol. 57, 1-18, (2015).

[23] W. Lu, J. Yang, X. Liu and W. Pedrycz, The modeling and prediction of time series based on synergy of high-order fuzzy cognitive map and fuzzy c-means clustering, Knowledge-Based Systems., vol. 70, 242-255, (2014).

[24] G. A. Miller, The magical number seven, plus or minus two: some limits on our capacity for processing information, Psychological Review., vol. 2, 343-352, (1994).

[25] S. Rubinstein, A. Goor and A. Rotshtein, Time series forecasting of crude oil consumption using neuro-fuzzy inference, Journal of Industrial and Intelligent Information., vol. 3, 84-90, (2015).

[26] H. J. Sadaei, R. Enayatifar, A. H. Abdullah and A. Gani, Short-term load forecasting using a hybrid model with a refined exponentially weighted fuzzy time series and an improved harmony search, International Journal Electrical Power and Energy Systems., vol. 62, 118129, (2014).

[27] Q. Song and B. S. Chissom, Fuzzy time series and its models, Fuzzy Sets and Systems., vol. 54, 269-277, (1993).

[28] B. Q. Sun, H. Guo, H. R. Karimi, Y. Ge and S. Xiong, Prediction of stock index futures prices based on fuzzy sets and multivariate fuzzy time series, Neurocomputing., vol. 151, 1528-1536, (2015).

[29] L. Wang, Y. Zeng and T. Chen, Back propagation neural network with adaptive differential evolution algorithm for time series forecasting, Expert Systems with Applications., vol. 42, 855-863, (2015).

[30] J. Wang and S. Xiong, A hybrid forecasting model based on outlier detection and fuzzy time series - A case study on Hainan wind farm of China, Energy., vol. 76, 526-541, (2014).

[31] H. C. Wu, Statistical confidence intervals for fuzzy data, Expert Systems with Applications., vol. 36, 2670-2676, (2009).

[32] L. A. Zadeh, Fuzzy sets, Information and Control., vol. 8, 338-353, (1965). 